\title{
The pandemic nature of reemerging tuberculosis and the role of population migration in its spread
}

\section{Background and history}

March 24 is the World TB Day which commemorates the date in 1882 when Robert Koch announced the discovery of Mycobacterium tuberculosis, the bacillus causing tuberculosis (TB). TB is a common and deadly infectious disease caused by gram-positive bacterial species from the Mycobacteriaceae family, Mycobacterium tuberculosis in humans or Mycobacterium bovis in cattle and sometimes humans in underdeveloped countries. TB spreads through air contaminated by droplets from infected people when they cough or sneeze. The disease affects mostly the lungs (pulmonary TB) but can also touch the central nervous system, lymphatic system, circulatory system, genitourinary system, bones, joints, and even the skin. Worldwide, TB remains one of the leading causes of death from infectious disease. Each year, approximately 9million persons around the world become ill with TB, and nearly 2 million TB-related deaths occur worldwide. ${ }^{1}$ The number of reported TB cases in the United States is at an all-time low, with 17 consecutive years of decline. However, racial/ethnic minority populations and foreign-born persons continue to account for a disproportionate percentage of TB cases. ${ }^{2}$ TB has been an affliction of humankind since before recorded history. More than 2 billion people, equal to one-third of the world's population, are infected with TB. 1 in 10 people infected with TB will become sick with active TB in their lifetime. The most common symptoms are severe weight loss, night sweats; chronic cough and coughing up of blood or blood stained sputum, known as hemoptysis. A person may be infected in the early decades of life and remain healthy and free of disease for decades. The infecting organisms remain viable; and the disease may be activated by malignancy, immune suppression, old age or chronic ill-health. There is a $10 \%$ life-long risk of developing disease (HIV/AIDS patients have a $10 \%$ annual risk of developing disease). The vast majority of TB deaths are in the developing world, and more than half of all deaths occur in Asia. TB incidence rates vary substantially from regions to regions and from countries to countries. In countries such as U.S. where TB incidence rate is low, increasing immigration trends may have significant impact on the TB transmission patterns in these countries. Crowded conditions, poverty, and host susceptibility facilitate the spread of this disease within populations. These situations have evolved over the past millennium and over the past decade affecting the trends in TB incidence in the United States, the rest of the world, and specific subpopulations. Anthropological data suggest that $M$. bovis may have caused ancient forms of tuberculosis. M. bovis was endemic to animals before humans evolved, and probably was transmitted from prey to predator. After humans evolved, $M$. bovis may have been transmitted in an analogous way, from animals killed for food. Active infections, which would be similar to tuberculosis as it is known today, probably remained sporadic and uncommon because $M$. bovis is poorly adapted to transmission between humans. Evidence for tuberculosis in ancient civilizations has come from the remains of ancient Egyptians, early Hindu writings referring to a disease called consumption, and ancient Greek medical literature referring to tuberculosis as phthisis. There is strong evidence that TB has plagued humankind since prehistory, and

\author{
Volume 6 Issue 4 - 2017
}

\author{
MichaelW Popejoy,' Javier Gasana, ${ }^{2}$ Rakesh \\ Ravikumaran, ${ }^{3}$ Daniel Westphal, ${ }^{4}$ Angelico \\ Mendy \\ 'Professor of Public Health, Florida International University, USA \\ ${ }^{2}$ Department of Health Promotion and Disease Prevention, \\ Florida International University, USA \\ ${ }^{3}$ Professor, Florida International University, USA \\ ${ }^{4}$ Chief Medical Officer, Broward Health, USA \\ ${ }^{5}$ Department of Epidemiology, The University of lowa, USA
}

Correspondence: MichaelW Popejoy, Professor of Public Health, Robert Stempel School of Public Health and Social Work, Florida International University, Florida, USA, Email dr_popejoy@hotmail.com

Received: July 04, 2017 | Published: November 09, 2017

the infection is believed to have claimed more lives than any other microbial pathogen. ${ }^{3}$

Investigations using genetic data suggest that progenitor species of Mycobacterium tuberculosis may have existed and infected hominids about 3 million years ago in East Africa. ${ }^{4}$ Skeletal deformities indicative of spinal infection by Mycobacterium tuberculosis (Pott's disease or tuberculosis Spondilytis) have been found in ancient Egyptian mummies dating back to $3400 \mathrm{BC}$. Molecular examinations of mycobacterial DNA from mummies have confirmed the osseous presence of Mycobacteria tuberculosis and bovis. ${ }^{5,6}$ Evidence of TB presence in India 3,300years ago, in China 2,300years ago, in classical Greece (where it was called phtisis), and Rome in the 5th century has also been reported. ${ }^{3}$ It was, however, only in the $19^{\text {th }}$ century that the pathogenesis of TB was elucidated with the work of the pathologist René Théophile Hyacinthe Laennec who described morbid anatomical changes of TB. ${ }^{7}$ In 1865 , TB was proven an infectious disease by Jean Antoine Villemin, an army surgeon who inoculated purulent liquid from a tuberculosis cavity to a rabbit who later died of the disease. ${ }^{8}$ Seventeen years later, Robert Koch, ${ }^{9}$ a German physician, discovered that Mycobacterium tuberculosis was the cause of TB, by isolating the bacteria from infected people, and later inoculating it to animals which developed the disease, and subsequently died from it. Robert Koch received the Nobel Prize in Medicine and Physiology in 1905 for this discovery. In 1907, an Austrian pediatrician, Cemens Freiherr von Pirquet developed the tuberculin skin test in the form of a vaccination to intracutaneously inject small quantity of diluted tuberculin. After extensive study of tuberculin reaction, a cut of diameter of $5 \mathrm{~mm}$ was set as a positive reaction. The use of cannulated needle and syringe as well as a purified protein derivative was soon introduced. ${ }^{10}$ In 1921, Albert Calmette and Camille Guerin developed the Bacille CalmetteGuerin (BCG) vaccine from an attenuated species of Mycobacterium bovis initially isolated by Nocard in 1902. Calmette and Guerin's work started in 1908, when they began series of cultures on glycerol-potato- 
bile medium; and in 1921, an attenuated mycobacterium for the use of a vaccine was obtained. The vaccine was first tested on an infant whose mother died of pulmonary tuberculosis. The child survived, and fortunately did not develop TB. ${ }^{11}$ However, it was not until 1943 that the first anti-TB drug, Streptomycin was discovered by Selman Wakman. The development of other drugs including pyrazinamide in 1952, cycloserine in 1952, ethionamide in 1956, rifampicine in 1957 and ethambutol in 1962. ${ }^{12}$

\section{Transmission of tuberculosis}

Mycobacteria are aerobic and nonmotile bacteria (except for the species Mycobacterium marinum, which has been shown to be motile within macrophages) that are characteristically acid-alcohol fast. ${ }^{13}$ Mycobacteria do not contain endospores or capsules, and are usually considered Gram-positive. There are more than 70 species of mycobacteria. Of these, two are major pathogens Mycobacterium tuberculosis was first isolated by Robert Koch; and Mycobacterium leprae was discovered by a Norwegian physician, Gerhard A Hansen in 1874. The remaining mycobacteria are environmental organisms, collectively known as MOTTs (Mycobacteria Other Than Tuberculosis). MOTT organisms are responsible for opportunistic infections, especially in people with AIDS. In nearly one-third of the world's population, the infection is dormant, held in check by the body's immune system. Such people are said to have latent TB infection; they exhibit no symptoms, and cannot spread the disease. However, in some people, the infection becomes active, and causes TB disease. People with active tuberculosis in their lungs can pass the bacteria on to anyone they come into close contact with. When a person with active tuberculosis coughs, sneezes or spits; people nearby may breathe in tuberculosis bacteria, and become infected. Left untreated, each person with active tuberculosis will infect on average between 10 to 15 people annually. People can also be infected with tuberculosis not active in the body. Inactive tuberculosis infection is called latent tuberculosis. If a person has latent tuberculosis, it means their body has been able to successfully fight the bacteria, and stop causing illness. People who have latent tuberculosis do not feel sick, do not have symptoms, and cannot spread tuberculosis.

In some people, tuberculosis bacteria remain inactive for a lifetime without becoming active. But in some other people the inactive tuberculosis may become active tuberculosis if their immune system becomes weakened; for example, by HIV. People with inactive tuberculosis are also called TB carriers. Tuberculosis infects the human host following inhalation of small infectious particles called droplet nuclei. Infectious droplet nuclei measure approximately 1 to 5 microns in diameter, deposit in the terminal bronchioles and alveoli of the host; and theoretically may contain as few as one viable organism. The bacteria may multiply briefly at the site of deposition but eventually are ingested by pulmonary macrophages. M. tuberculosis interacts with macrophages mainly via complement receptors; but, with mannose receptors as well in a cooperative manner. Following phagocytosis, $M$. tuberculosis inhibits phagolysosomal fusion, allowing the organism to survive, and multiply intracellularly within lysosomes. The specific attenuation of the cellular immune response; which allows continuous intracellular survival of the organism, is still not well understood. The M. tuberculosis organisms can remain latent within the reticuloendothelial system both within pulmonary; and extra pulmonary sites. The droplet nuclei, can be generated when persons with infectious TB sneeze, cough, speak or sing. The particles are so small that normal air currents can keep them airborne for hours; and spread them throughout a room or building. Infection occurs when a susceptible person inhales droplet nuclei containing TB bacteria, and the bacteria become established in the lungs, and then spread throughout the body. Transmission of tuberculosis to other human hosts is strictly via droplet nuclei. M. tuberculosis within secretions; or droplet nuclei that have deposited on a surface, reduce the potential for infection. Patients with pulmonary or laryngeal TB produce infectious droplet nuclei. Those with extra pulmonary tuberculosis do not, unless the site of TB infection is manipulated in such a way that an aerosol is generated. Transmission of infection to another human host is generally a function of the concentration of infectious droplet nuclei, duration of contact with the infectious case, and the susceptibility of the host exposed. Data from the U.S. Centers for Disease Control and Prevention (CDC) show that approximately $21 \%$ to $23 \%$ of individuals in close contact to patients with infectious tuberculosis become infected. Two to ten weeks after initial infection, the body's immune response usually limits further multiplication and spread of TB bacteria. However, in a small proportion of cases, (usually less than $1 \%$ ), initial infection rapidly progresses to clinical illness or active TB. For another group (approximately 5\%-10\%), active TB develops after an interval of months, years or decades. The risk of progression to active TB is markedly increased for persons infected with the human immunodeficiency virus (HIV), the causative agent of AIDS.

An infectious person coughs about 1 to 3 airborne organisms. The organism remains suspended in the air indefinitely which is inhaled by the person being infected, and are taken up by alveolar macrophages. One organism may be enough to establish an infection. Specific characteristics can predict when a patient with TB will be more likely to transmit the disease to others, although the science is not exact. Cavitary disease increases the probability of infection among contacts because of the large number of organisms in the sputum from these patients. A study from Finland suggested the probability of active tuberculosis was higher among contacts of patients who produced sputum smears containing high numbers of organisms. At the other end of the spectrum, patients who produce low concentrations of organisms in sputum; those who are smearnegative, but culture-positive, are the least likely to transmit infection; yet transmission does occur at low levels. When a patient with active pulmonary disease cooperates in covering their nose and mouth when coughing or sneezing, or in wearing an ordinary surgical mask, the large droplets with the potential to form infectious droplet nuclei are captured and inactivated. The effect of a physical barrier rather than the filtration properties is what is important with such techniques. The volume of air common to the source and the contact is one such factor. The smaller the room, the more concentrated the droplet nuclei. Indeed, TB incidence in NZ is associated with household crowding. ${ }^{14}$

Reducing or eliminating household crowding could decrease TB incidence in NZ and globally. The amount of outside air ventilated into a room is another factor since fresh air will dilute the number of droplet nuclei. Modem buildings are engineered for air recirculation. Closed heating and air conditioning systems increase the concentration of droplet nuclei since not much outside air is introduced into such a system. Engineering controls that reduce contamination include passage of recirculated air across a UV light source or across highefficiency particulate air (HEPA) filters. More exposure for longer duration means the greater the probability of inhaling critical numbers of droplets exceeding the threshold for infection. Naive hosts who are immunosuppressed or at the extremes of age (under 5 or over 65) are 
more likely to become infected when they are in close contact with a patient with a positive sputum smear. In contrast; close contacts with those who have been infected previously, demonstrable by a positive PPD skin test, are very unlikely to be reinfected as long the immune and health status is intact.

\section{Tuberculosis and human immunodeficiency virus (HIV)}

$\mathrm{TB}$ is a disease spread from person to person through the air This disease is particularly dangerous for persons infected with HIV. Worldwide, TB is a leading cause of death among persons infected with HIV. ${ }^{15}$ Because it is spread through the air, the increase in active tuberculosis among people infected with both tuberculosis and HIV results in:

\section{i. More transmission of the tuberculosis bacteria, \\ ii. More people with latent tuberculosis, \\ iii. More TB disease in the population.}

People with latent tuberculosis are increasingly becoming infected with HIV, and many more are developing active TB because HIV is weakening their immune system. People who are co-infected with both HIV and latent TB have an up to 800 times greater risk of developing active tuberculosis disease, and becoming infectious compared to people not infected with HIV. People with advanced HIV infection are vulnerable to a wide range of infections and malignancies that are called "opportunistic infections" because they take advantage of the opportunity offered by a weakened immune system. Tuberculosis is an HIVAIDS related opportunistic infection. A person that has both HIV and active tuberculosis has a defining illness. There are several important associations between the epidemics of HIV and tuberculosis:

\section{i. Tuberculosis is harder to diagnose in HIV positive people,}

ii. Tuberculosis progresses faster in HIV-infected people,

iii. Tuberculosis in HIV positive people is more likely to be fatal if undiagnosed or left untreated,

iv. Tuberculosis occurs earlier in the course of HIV infection than other opportunistic infections,

v. Tuberculosis is the only major AIDS-related opportunistic infection that poses a risk to HIV-negative people.

WHO and its international partners have formed the TB/HIV Working Group, which develops global policy on the control of HIVrelated TB and advises on how those fighting against TB and HIV can work together to tackle this lethal combination. The interim policy on collaborative TB/HIV activities describes steps to create mechanisms of collaboration between TB and HIV/AIDS programmes, to reduce the burden of TB among people and reducing the burden of HIV among TB patients.

\section{TB as a pandemic}

Many people may think of tuberculosis as being a disease from the past. The TB incidence rate changed annually within a range of $+/-10 \%$ over the study period in the 134 countries examined, and its average value declined in 93 countries. ${ }^{16}$ The rate was declining more quickly in countries that had a higher human development index, lower child mortality, and access to improved sanitation.
General development measures were also dominant explanatory variables within regions, though correlation with TB incidence trends varied widely geographically. The TB incidence rate was falling more quickly in countries with greater health expenditure (situated in central and eastern Europe and the eastern Mediterranean), highincome countries with lower immigration, and countries with lower child mortality and HIV infection rates (located in Latin America and the Caribbean).Tuberculosis is mutating into dangerous new strains for which there is no known cure. There were 9.4million new TB cases in 2008 (3.6million of whom are women) including 1.4million cases among people living with HIV. More than 1.8 million died from TB in 2008, including 500,000 people with HIV-equal to 4,500 deaths a day. The majority of cases are found in Asia and Africa. The estimated global incidence rate fell to 139 cases per 100000 population in 2008 after peaking in 2004 at 143 cases per 100000 . Rates are falling very slowly in 5 WHO regions (the rate is stabilizing in Europe). The total number of deaths and cases is still rising due to population growth. TB is a leading killer of people with HIV. People who are HIV-positive and infected with TB are 30 times more likely to develop active TB than people not infected with HIV living in the same country. TB is responsible for $25 \%$ of adult deaths in the developing world-more than those caused by diarrhea, malaria and AIDS combined. South Africa has one of the highest incidences with more than 200 per 100,000. In Ravensmead near Cape Town there are more than 400 cases per year. The total population of that suburb is 40000 people. 35 people die of TB every day in South Africa. The incidence in the developed countries is less than $10 / 100000 .^{17}$

There were more than 800,000 cases of leprosy worldwide in 1998, these being non-tuberculosis mycobacterial infections; of these more than 690,000 were new cases. In the U.S., environmental mycobacteria are isolated more frequently than M. tuberculosis from clinical specimens; this is due to their association with AIDS, and the low incidence of TB. Less than 3\% of mycobacterial isolates in South Africa are of MOTT organisms. Buruli Ulcer is the third most common mycobacterial infection, and is endemic in West Africa. However, more than 25,000 people contract it every year in the United States. For 2009, a total of 11,540 tuberculosis (TB) cases were reported in the United States. The TB rate was 3.8 cases per 100,000 population; a decrease of $11.4 \%$ from the rate of 4.2 per 100,000 reported for 2008 . The 2009 rate showed the greatest single-year decrease ever recorded and was the lowest recorded rate since national TB surveillance began in $1953 .{ }^{2}$ So, $12,904 \mathrm{~TB}$ cases (a rate of 4.2 cases per 100,000 persons) were reported in the United States in 2008. Both the number of TB cases reported and the case rate decreased; this represents a $2.9 \%$ and $3.8 \%$ decline, respectively, compared to 2007 . The TB rate in 2008 was the lowest recorded since national reporting began in 1953. The TB rate has been going down in the United States each year since 1992. However, progress has slowed in the recent years. The average annual percentage decline in the TB rate slowed from $6.6 \%$ for 1993 through 2002, to an annual average decline of $3.4 \%$ for 2003 through 2008. In 2008, the TB rate in foreign-born persons in the United States ( 20.3 cases per 100,000 persons) was 10 times greater than that of USborn persons ( 2.0 cases per 100,000 persons).

Also of note, $59 \%$ of all TB cases in the United States occurred in foreign-born persons in 2008. There were 644 deaths from TB in 2006, the most recent year for which these data are available. Compared to 1996 data, when 1,202 deaths from TB occurred, this represents a $46 \%$ decrease in TB deaths in the last decade. Based on surveillance and survey data, WHO estimates that 9.27 million new cases of TB 
occurred in 2007 (139 per 100000 population), compared with 9.24million new cases (140 per 100000 population) in $2006 .{ }^{17}$ Of these 9.27 million new cases, an estimated $44 \%$ or 4.1 million (61 per 100000 population) were new smear-positive cases. India, China, Indonesia, Nigeria and South Africa rank first to fifth in terms of the total number of incident cases. Asia (the South-East Asia and Western Pacific regions) accounts for 55\% of global cases and the African Region for $31 \%$; the other three regions (the Americas, European and Eastern Mediterranean regions) account for small fractions of global cases. ${ }^{17}$ Indeed, TB is a disease of poverty affecting mostly young adults in their most productive years. The estimated incidence rate in sub-Saharan Africa is nearly twice that of the South-East Asia Region, at nearly 350 cases per 100000 population. Both the highest number of deaths and the highest mortality per capita are in the Africa Region. The TB epidemic in Africa grew rapidly during the 1990s, but this growth has been slowing each year, and incidence rates now appear to have stabilized or begun to fall. In 2005, estimated per capita TB incidence was stable or falling in all six WHO regions. However, the slow decline in incidence rates per capita is offset by population growth. Consequently, the number of new cases arising each year is still increasing globally and in the WHO regions of Africa, the Eastern Mediterranean and South-East Asia.

\section{TB among various groups}

\section{Diverse populations}

In 2009, a total of 4,499 TB cases were reported in US-born persons (representing $39.8 \%$ of the 11,305 cases with known national origin), compared with 5,282 reported in $2008 .^{2}$ The 2009 rate in USborn persons was 1.7 per 100,000 , a decrease of $15.8 \%$ compared with 2008, and a decrease of $77 \%$ compared with 1993 . In 2009, a total of 1,861 cases were reported among blacks, representing the highest number of TB cases among US-born persons and $41.4 \%$ of all US-born cases in 2009. A total of 3,386 cases were reported among Hispanics, more than any other racial/ethnic group, followed by Asians and blacks. Asians had the highest TB case rate among all racial/ethnic groups. From 2008 to 2009, TB rates decreased for all racial/ethnic minorities. The greatest annual decrease in TB rate was among whites (15.2\%), followed by blacks (14.0\%) and Hispanics (13.6\%). The smallest decrease in 2009 was among Asians (9.0\%). TB in blacks was associated with younger age, inner city residence, HIV seropositivity, and drug resistance. ${ }^{18}$ The slowing decline in TB incidence and persistent disparities between US-born and foreignborn persons and between whites and minorities threaten progress toward TB elimination in the United States. ${ }^{19}$ A total of $6,806 \mathrm{~TB}$ cases were reported in foreign-born persons in 2009, compared with 7,602 reported in 2008 , a decrease of $10.5 \% .^{2}$

For 2009, these cases represented $60.2 \%$ of all cases with known national origin, compared to $59.0 \%$ of cases with known origin in 2008. The TB rate among foreign-born persons in 2009 was 18.6 per 100,000 ; a $9.0 \%$ decrease compared to 2008 and a $45.3 \%$ decrease since 1993. In 2009, four countries accounted for $50.1 \%$ of TB cases in foreign-born persons: Mexico (1,574), the Philippines (799), India (523), and Vietnam (514). Intensified efforts are needed to address the slowing decline in TB incidence and the persistent disparities that exist between US-born and foreign-born persons and between whites and minorities in the United States. ${ }^{2}$ In 2009, among persons with TB with a known human immunodeficiency virus (HIV) test result, $10.2 \%$ (690 of 6,743) were co-infected with HIV. Part of the 2009 decrease in foreign-born cases also might be attributed to new technical instructions issued in $2007^{20}$ for pre-immigration TB screening. These instructions might have prompted more TB diagnoses and treatment before immigration because, for the first time, they require culture of respiratory specimens in immigrants and refugees who are suspected of having pulmonary TB based on chest radiographs. The previous technical instructions only required smear microscopy, which is less sensitive and has been shown to result in fewer TB diagnoses. Since 1993, when the TB surveillance system was expanded to include drug-susceptibility results, reported multidrug-resistant (MDR) TB cases have decreased in the United States. The most recent year for which complete drug-susceptibility data are available is $2008 .{ }^{21}$ Drugsusceptibility test results for isoniazid and rifampin were reported for $96.0 \%(9,628$ of 10,034$)$ of culture-confirmed TB cases in 2008 , compared with $97.9 \%(10,251$ of 10,468$)$ in 2007 . A total of 107 cases of multidrug-resistant TB (MDR TB) were reported in 2008, which represented $1.1 \%$ of all culture-positive cases with drug-susceptibility testing, compared with $1.2 \%$ [123 of 10,251 cases] in 2007. For persons with a previous history of $\mathrm{TB}$, the percentage of cases with MDR TB remained approximately four times higher than for persons without a prior history of TB. In 2008, foreign-born persons accounted for $77.6 \%$ of MDR TB cases.

Foreign-born persons had higher percentages of MDR TB than US-born persons, both among persons with $(4.3 \%$ versus $1.4 \%)$ and without $(1.0 \%$ versus $0.4 \%)$ a previous history of TB. To date, no new cases of extensively drug-resistant TB (XDR TB) have been reported in 2009. With regard to tuberculosis, health disparities persist among members of racial and ethnic minority populations. In 2007, the majority ( $83 \%$ ) of all reported TB cases in the United States occurred in racial and ethnic minorities. ${ }^{22}$ Black, non-Hispanic persons, have a disproportionate share of TB in the US. In 2007, TB was reported in 3,470 black, non-Hispanic persons, $26 \%$ of all persons reported with TB nationally. Also, in 2007, the rate of TB in black, nonHispanic persons was 9.4 cases per 100,000 populations, which is approximately 8.5 times higher than the rate of TB in white, nonHispanic persons (1.1 cases per 100,000 populations). The proportion of TB in black, non-Hispanic persons is even greater if only USborn (African American) persons reported with TB are examined. In 2007, among US-born persons reported with TB, $45 \%$ were African Americans (black, non-Hispanic). Although rates of TB in both blacks and whites have declined substantially over the past decade, the disparity remains. We must better target our efforts to prevent and control TB in this population. Addressing the TB disparity among African Americans and other US-born racial/ethnic groups is an important priority. Both census-based indicators performed well in distinguishing areas with high TB incidence rates from areas with little or no TB. ${ }^{23}$ Due to simplicity, the single poverty measure rather than the multifactorial Townsend index might be especially useful in identifying high-risk neighborhoods for targeted TB prevention efforts. In many developing countries, TB is a serious international public health problem. Although MDR and extensively drug-resistant (XDR) TB are occurring globally, they are still rare. HIV-infected travelers are at greatest risk if they come in contact with a person with MDR or XDR TB. All travelers should avoid high risk settings where there are no infection control measures in place. Documented places where transmission has occurred include crowded hospitals, prisons, homeless shelters, and other settings where susceptible persons come in contact with persons with TB disease. 
Air travel itself carries a relatively low risk of infection with TB of any kind. Untreated TB represents a greater hazard to a pregnant woman and her fetus than does its treatment. Treatment of pregnant women should be initiated whenever the probability of TB is moderate to high. Infants born to women with untreated TB may be of lower birth weight than those born to women without TB and, rarely, the infant may be born with TB. Although the drugs used in the initial treatment regimen cross the placenta, they do not appear to have harmful effects on the fetus. Breastfeeding should not be discouraged for women being treated with the first-line anti-tuberculosis drugs because the concentrations of these drugs in breast milk are too small to produce toxicity in the nursing newborn. For the same reason, drugs in breast milk are not an effective treatment for TB disease or LTBI in a nursing infant. Breastfeeding women taking INH should also take pyridoxine (vitamin B6) supplementation.

\section{Tuberculosis and overcrowding in correctional institutions}

Ill-health thrives in settings of poverty, conflict, discrimination and disinterest. ${ }^{24}$ Prison is an environment that concentrates precisely these issues. TB is a major cause of sickness and death in prisons, along with HIV, malnutrition, mental illness and violence. Directly or indirectly, these threats apply, not only to prisoners, but to all who come into contact with prisons and ultimately the community as a whole. Prisoners very often originate from the most vulnerable sectors of society-the poor, the mentally ill, those dependent on alcohol or drugs. These groups already have an increased risk of diseases such as TB. In prison, these problems are amplified by poor living conditions and overcrowding. A prison climate of violence and humiliation aggravates the situation, creating obstacles in accessing health care and promoting unhealthy behaviors. Prisoners are eventually released back into society, bringing with them the illness and behaviors generated and worsened by their incarceration. More people are in jail, and recidivism occurs at a greater rate, than ever before. The number of people incarcerated increased from 1 of every 453 people in 1980 to 1 of 189 in 1994 . The rate of recidivism in 1994 was 61 percent. A correctional institution is a congregate setting that is ideal for transmission of tuberculosis between inmates and/or the correctional workers. Also, evidence indicates that prisoners released into the communities extend transmission, particularly to children in the home.

Tuberculosis is more prevalent in prison populations compared to its prevalence in the general population. Fourteen to 25 percent of inmates have a positive PPD skin test. The probability of tuberculosis infection increases directly with the length of incarceration, which indicates that transmission of TB must occur in prisons. The rate of tuberculosis in prisoners compared with that in the general population varies depending on the prison location. For example, in New York State, the rate of TB in the prisons is 6.3 times the rate for that state's general population, whereas in New Jersey and California the values are even higher at 11 and 10-fold, respectively. Many factors explain why there are high rates of tuberculosis in correctional institutions. Many state and federal facilities operate up to $25 \%$ above design capacity. Overcrowding, coupled with typically poor ventilation in the prison environment, facilitates aerosol transmission of tuberculosis. The high rate of HIV infection is another factor, which is highlighted by a recent study showing that the HIV-seropositive rate in the prison population was approximately 50 times greater than that in a matched population of military recruits.
In the absence of HIV infection, history of intravenous drug abuse is associated with a higher risk of tuberculosis. In a survey of 20,000 state and federal prisoners from 45 states, 25 percent of the inmates had a history of IV drug abuse. Also, the prison population represents a lower socioeconomic group, a segment of the population that is more commonly infected with tuberculosis. All of these factors taken together help explain the higher rates of tuberculosis among individuals housed in correctional institutions. TB control can be particularly problematic for correctional facility staff and inmates in correctional and detention facilities, in which persons from diverse backgrounds and communities are housed in close proximity for varying periods. Effective TB prevention and control measures in correctional facilities include:

i. Early identification of persons with TB disease through entry and periodic follow-up screening.

ii. Successful treatment of TB disease and latent TB infection.

iii. Appropriate use of airborne precautions (e.g., airborne infection isolation, environmental controls, and respiratory protection).

iv. Comprehensive discharge planning.

v. Thorough and efficient contact investigation.

These measures should be instituted in close collaboration with local or state health department TB-control programs and other key partners. Continuing education of inmates, detainees, and correctional facility staff is necessary to maximize cooperation and participation. To ensure TB prevention and control measures are effective, periodic program evaluation should be conducted. Tuberculosis facilities should be used to set up social centers for the complex work and treatment of socially dysadapted patients with tuberculosis (including those who have been released from confinement institutions). ${ }^{25}$ For this, tuberculosis institutions should be staffed by social workers, psychologists, psychiatrists, lawyers, and narcology experts with the appropriate funds being allocated.

\section{Tuberculosis in IV drug abusers}

Tuberculosis incidence rates have been rising predominantly among the poor, minority, and intravenous drug-using populations. Congregate housing settings such as city shelters, nursing homes, jails, and prisons provide ideal opportunities for transmission of TB. The IV drug abuse population has a higher incidence of tuberculosis than does the general population in areas of the United States where tuberculosis is prevalent. Higher rates of HIV co-infection within the IV drug abuse population increase the risk of a tuberculosis infection and the development of active disease in this population.

\section{Tuberculosis in the elderly}

TB in older people does not follow a predictable course. It is often the reason that many physicians overlook the seriousness of the problem. The most common symptom is a chronic cough that may have had its origins with what appeared to be an ordinary cold or an episode of grippe or "flu." Loss of appetite and loss of weight often follow, too often attributed to poor eating habits, especially among those hampered by ill-fitting dentures or gum disease. Swallowing difficulties in the elderly are also implicated in diagnosing weight loss and reduction of food intake. Analogous to that in the foreign-born population, the majority of tuberculosis cases in the elderly population are a result of activation of a prior infection, and only approximately 10 to 20 percent of active cases are due to primary infection. 


\section{Transmission of tuberculosis during airline flights}

The World Health Organization (WHO)'s international guideline for the control of tuberculosis in relation to air travel-tracing passengers who sat for longer than eight hours in rows adjacent to people with pulmonary TB-may be futile and exaggerated. A British scientist who reviewed evidence from 39 studies that looked at transmission of TB during commercial air travel found that there was little risk of TB transmission linked to air journeys. The risk of $M$. tuberculosis transmission to other passengers during airline flight is not greater than in any other confined spaces. Several studies have even shown that passengers with documented cavitary pulmonary disease did not infect other passengers.

\section{Nosocomial transmission of tuberculosis}

\section{Introduction}

Recent population data reveals that 400 million humans are on the move globally as displaced refugees flee civil unrest and war in their home nations. When humans migrate, so do the human diseases that they bring with them as they travel across international boundaries to safe havens fleeing war or civil unrest. TB is a worldwide disease, and nosocomial transmission is known to occur. The major causes of tuberculosis transmission within hospitals are from those cases where it is not suspected, the diagnosis is delayed, or the respiratory isolation procedure breaks down. Medical students, pathologists, and assistants working in an autopsy room exhibit a higher risk for tuberculosis infection and active disease.

The autopsy suite stands out as one of the hospital sites where the heaviest exposure to tuberculosis may occur for several reasons; when cutting infected lung or bone with a knife or oscillating saw, an aerosol with a high density of bacteria is likely to be generated. Recent data show that the concentration can be as high as 1 infectious unit per 3.5 cubic feet of air, a far more dense concentration when one considers that on a tuberculosis ward the concentration measures approximately 1 infectious unit per 24.000 cubic feet of air. Also, autopsy workers are more frequently exposed to patients unsuspected of having tuberculosis ante mortem, so adequate respiratory protection may not be in place. As an example, the extensive MDRTB outbreaks that occurred in eight hospitals and a New York state prison illustrated several common characteristics of nosocomial transmission. Delayed diagnosis, delay in effective treatment, lack of effective isolation procedures, and a high proportion of patients with severe AIDS $\left(\mathrm{CD}^{4+}\right.$ lymphocytes less than $100 / \mathrm{mL}$ ) were all common features.

\section{Guidelines for protection of health care workers}

The current challenge is to develop an effective tool that will be beneficial locally and yet remain consistent with established scientific principles. At least four elements are needed for a successful local program:

i. Integrating important principles from existing guidelines.

ii. Collecting local epidemiologic data.

iii. Taking into account local capabilities and priorities.

iv. Ongoing monitoring for efficacy.

The resurgence of tuberculosis, and in particular the lessons learned from the MDRTB outbreaks, drove the process for reevaluating the 1990 CDC guidelines for tuberculosis containment in the hospital environment. These efforts culminated in detailed, broad guidelines published by the CDC and the National Institute for Occupational Safer and Health (NIOSH) at the end of 1994. The guidelines encompass three specific areas: administrative controls, environmental controls, and respiratory protection. The recommendations also recognize the geographic variation in TB prevalence and that the majority of hospitals in the United States are not faced with an overwhelming number of new cases. Therefore, a risk assessment for tuberculosis transmission is recommended for each institution. The level of risk classification is associated with a hierarchy of recommendations for control steps. The administrative and environmental controls portion of the guidelines recognizes the value of applying the traditional tuberculosis infection control practices.

The development of a written tuberculosis control policy that includes specific plans for early identification, treatment, and isolation of patients with infectious tuberculosis are examples of issues addressed under the administrative controls portion of the guidelines. The engineering control recommendations are aimed at reducing the concentration of droplet nuclei within the patient's room. They focus primarily on mechanisms for negative-pressure ventilation in hospital rooms.

\section{TB Diagnosis}

\section{Tuberculin}

There is great excitement in the TB scientific community over the introduction of new tools into TB control activities. The development of new tools is an important component of the Global Plan to Stop TB and the World Health Organization's new global Stop TB Strategy. However, TB can be diagnosed by injecting a protein found in TB bacteria into the skin of an arm. If the skin reacts by swelling then the person is probably infected with tuberculosis. However, this method is not wholly reliable at detecting TB infections among HIV-infected people because their weakened immune systems often cannot mount a strong enough defense against the injected proteins to cause swelling. It also detects both active and latent tuberculosis, meaning the test is not very accurate at diagnosing active TB disease in people who live in areas where tuberculosis (and thus latent TB infection) is very common. The size of a positive test result depends on the exposure history and health status of the individual and a measure of the actual in duration rather than the erythema produced. ${ }^{26}$ Tuberculin is the name given to extracts of Mycobacterium tuberculosis, M. bovis, or M. aviumthat is used in skin testing in animals and humans to identify a tuberculosis infection. Several types of tuberculin have been used for this, of which purified protein derivative (PPD) is the most important. PPD evokes a delayed hypersensitivity (DTH) response when injected into the skin and this forms the basis of the Tuberculin Skin Test. Tuberculin skin testing can be used to identify individuals, especially children, with active tuberculosis. It can be used to trace contacts of patients with active tuberculosis.

A positive tuberculin test may be an indication for INH prophylaxis. Diagnosis of tuberculosis in the lungs may be made using an X-ray or sputum test, but again, these may not give a clear indication of active TB infection in HIV positive people, because their immune systems are not strong enough to mount an inflammatory reaction against the bacteria. In cases of extra-pulmonary tuberculosis (where the disease is affecting organs other than the lungs), fluid or tissue samples may be tested. If there is doubt about the diagnosis of tuberculosis, a culture of TB bacteria can also be grown in a laboratory. However, this requires 
specialized and costly equipment and can take six to eight weeks to produce a result. If the necessary facilities are unavailable then the tuberculosis diagnosis is often based on symptoms.

\section{U.S. CDC guidelines for evaluating chest X-ray (CXR)}

An anterior-posterior (AP) chest X-ray is the standard view used; other views (lateral or lordosis) or CT scans may be necessary. In active pulmonary $\mathrm{TB}$, infiltrates or consolidations and/or cavities are often seen in the upper lungs with or without mediastinal or hilar lymphadenopathy. ${ }^{27}$ However, lesions may appear anywhere in the lungs. In HIV and other immuno suppressed persons, any abnormality may indicate TB or the chest X-ray may even appear entirely normal. Old healed tuberculosis usually presents as pulmonary nodules in the hilar area or upper lobes, with or without fibrotic scars and volume loss. Bronchiectasis and pleural scarring may be present. A medical examination is mandatory for all refugees coming to the US and all applicants outside the US applying for an immigrant visa.

The purpose of the medical examination is to identify applicants with inadmissible health-related conditions such as active tuberculosis. Outside the US, medical examinations are performed by approximately 400 physicians (panel physicians) selected by United States Department of State consular officials. In the US, medical examinations are performed by approximately 3,000 physicians (civil surgeons) designated by district directors of the US Citizenship and Immigration Services. Guidelines were developed by the Centers for Disease Control and Prevention (CDC). The chest X-ray and classification worksheet is designed to group findings into categories based on their likelihood of being related to TB or non-TB conditions needing medical follow-up (either at the time of the chest X-ray or after resettlement).

\section{Bacteriologic examination}

Bacteriologic examination of specimens such as sputum, urine, cerebrospinal fluid is of major importance in the diagnosis of TB. People suspected of having pulmonary TB should have sputum specimens collected three times, each in 8 to 24 hours intervals, and tested for Mycobacterium. There are four methods for specimen collection for pulmonary TB. Coughing is the most common method to extract sputum up from the lung. Other methods to collect specimens for pulmonary TB include sputum induction (sputum producing coughing induced by inhalation of warm hypertonic saline), bronchoscopy (bronchoscopy may allow to collect bronchial washings, brushings, biopsy specimens), and gastric aspiration (to recover sputum coughed into the throat then swallowed).

For extra pulmonary TB, other specimen (urine, pus, cerebrospinal fluid, etc.) may be used in place of sputum. Initial bacteriologic evidence of mycobacterium can be obtained from microscopic detection of acid-fact bacilli in stained and acid-washed smears using Carbolfuchsin methods or Fluochrome procedure. It is estimated that 5,000 to 10,000 bacilli/milliter of specimen are needed to detect mycobacteria in stained smears, while only 10 to 100 are sufficient for a positive culture which is the gold standard. However, TB can be in diagnosed in the absence of positive $M$. tuberculosis culture, based on clinical symptoms and radiologic evidence. Broth-based system cultures may detect mycobacteria in 4 to 14days, while solid media may take 3 to 6 weeks. Direct detection using nucleic acid amplification can be used to DNA and RNA fragments from specimens to detect $M$. tuberculosis in only a few hours. M. tuberculosis found in specimens need to be tested for resistance to first anti-TB drugs. ${ }^{28}$

\section{TB Vaccine}

Bacille Calmette-Guerin (BCG) is the current vaccine for tuberculosis. BCG is the only vaccine available today for protection against tuberculosis. It is most effective in protecting children from the disease. An M. bovis strain was continuously sub cultured by Calmette and Guerin from 1908 to 1922 to produce the live attenuated strain named for them, bacillus Calmette-Guerin (BCG). BCG has been used as the basis for the live attenuated vaccine against tuberculosis since 1922. BCG is still the best available TB vaccine today and is used in many parts of the world. Many foreign-born persons have been BCG-vaccinated. BCG is used in many countries with a high prevalence of $\mathrm{TB}$ to prevent childhood tuberculosis meningitis and miliary disease. However, BCG is not generally recommended for use in the United States because of the low risk of infection with Mycobacterium tuberculosis, the variable effectiveness of the vaccine against adult pulmonary $\mathrm{TB}$, and the vaccine's potential interference with tuberculin skin test reactivity. The BCG vaccine should be considered only for very select persons who meet specific criteria and in consultation with a TB expert. The tuberculin skin test (TST) and blood tests to detect TB infection are not contraindicated for persons who have been vaccinated with BCG. BCG vaccination may cause a false-positive reaction to the TST, which may complicate decisions about prescribing treatment. The presence or size of a TST reaction in persons who have been vaccinated with BCG does not predict whether $\mathrm{BCG}$ will provide any protection against $\mathrm{TB}$ disease. Furthermore, the size of a TST reaction in a BCG-vaccinated person is not a factor in determining whether the reaction is caused by latent TB infection (LTBI) or the prior BCG vaccination. Blood tests to detect TB infection, unlike the TST, are not affected by prior BCG vaccination and are less likely to give a false-positive result.

\section{Treatment of tuberculosis}

\section{Current approach}

Today, doctors treat most people with TB outside the hospital. Gone are the days of going to the mountains for long periods of bed rest. Treatment of tuberculosis requires identifying patients with active disease from those with a latent infection. The current approach to treatment of active TB in particular, reflects the evolving epidemiology of tuberculosis. More emphasis is placed on ensuring adherence to treatment to head off the development of secondary resistance. Also, the recommendations for using INH to prevent a latent infection from progressing to active disease, and for using BCG vaccination as primary prevention, have both been updated in the last decade.

\section{Treatment of active tuberculosis}

Doctors will prescribe several special medications that you must take for six to nine months. Treatment takes that long because the disease organisms grow very slowly and, unfortunately, also die very slowly. The basic principles of therapy are to provide a safe, costeffective medication regimen in the shortest period of time. Multiple drugs are used in the initiation phase of treatment to rapidly reduce the number of viable organisms. Also, steps are taken to ensure adherence to treatment. To treat pulmonary and most forms of extra pulmonary tuberculosis in non immuno-suppressed patients as well as those coinfected with HIV, four of the following five first-line medications are used during the first 2months: Isoniazid, Rifampin, Pyrazinarnide, Ethambutol, or Streptomycin. Ethambutol or Streptomycin need not be given if the probability of resistance is extremely low (less than 
4 percent in the regional population) or susceptibility data indicates that the organism is susceptible to all first-line medications. Following the multi-drug induction phase, INH and Rifampin are given for an additional 4 months. This four-medication regimen has been shown to be highly effective. CDC data for the United States indicate that 95 percent of patients treated by this regimen will receive at least two drugs to which the infecting organism is susceptible. Also, patients who default before completing this regimen are more likely to be cured than those receiving fewer medications at the onset.

\section{Treatment for LTBI in BCG-vaccinated persons}

Using an adaptation of the U.S. Public Health Service (USPHS) rating system, CDC and ATS have rated LTBI treatment regimens based on the strength of recommendation and the quality of the evidence that supports that recommendation. Treatment of LTBI substantially reduces the risk that TB infection will progress to disease. Careful assessment to rule out the possibility of TB disease is necessary before treatment for LTBI is started. Evaluation of TST reactions in persons vaccinated with $B C G$ should be interpreted using the same criteria for those not BCG-vaccinated. Persons in the following high-risk groups should be given treatment for LTBI if their reaction to the TST is at least $5 \mathrm{~mm}$ of indurations or they have a positive result using a TB blood test:

\section{i. HIV-infected persons.}

ii. Recent contacts to a TB case.

iii. Persons with fibrotic changes on chest radiograph consistent with old TB.

iv. Patients with organ transplants.

v. Persons who are immuno suppressed for other reasons (e.g., taking the equivalent of more than $15 \mathrm{mg}$ /day of prednisone for 1month or longer, taking TNF-alpha antagonists).

In addition, persons in the following high-risk groups should be considered for treatment of LTBI if their reaction to the TST is at least $10 \mathrm{~mm}$ of in duration or they have a positive result using a TB blood test:

i. Recent arrivals (less than 5years) from high-prevalence countries.

ii. Injection drug users.

iii. Residents and employees of high-risk congregate settings (e.g., correctional facilities, nursing homes, homeless shelters, hospitals, and other health care facilities).

iv. Mycobacteriology laboratory personnel.

v. Persons with clinical conditions that place them at high-risk for developing TB disease (e.g., diabetes).

vi. Children less than 4years of age, or children and adolescents exposed to adults in high-risk categories.

Persons with no known risk factors for TB may be considered for treatment of LTBI if their reaction to the tuberculin test is at least $15 \mathrm{~mm}$ of indurations or they have a positive result using a TB blood test. Targeted skin testing programs should only be conducted among high-risk groups. All testing activities should be accompanied by a plan for follow-up care for persons with TB infection or disease. The modern approach to tuberculosis treatment incorporates interventions aimed at minimizing some of these problems so that patient adherence to therapy is improved. Supervised or directly observed therapy (DOT) is at the core of most of the more successful approaches. The advantages of DOT have been proven in several studies.

\section{Treatment of latent tuberculosis}

If a person with latent TB does not take INH or if s/he stops taking your pills too soon, s/he could become sick with active TB disease. This can happen to anyone with latent TB infection at any time. The indications for treatment of latent tuberculosis are based on the relative risk for progression to active disease balanced against the risk of INH hepatotoxicity, which increases with patient age, pre-existing liver disease, or the concurrent ingestion of certain medications and/ or alcohol. INH daily for 6 to 9 months is 65 to 80 percent effective in preventing a non immunosuppressed individual from developing active tuberculosis. Preventive INH treatment in an HIV patient with a latent infection also reduces the risk of developing active TB from 4.7 cases per 100 patient-years to 1.6 cases per 100 patient-years.

\section{Drug-resistant TB}

The 2008 WHO report said that there were 440,000 cases of multi drug resistant tuberculosis, with about one-third of them being fatal. Until 50 years ago, there were no medicines to cure TB. Now, strains that are resistant to a single drug have been documented in every country surveyed; what is more, strains of TB resistant to all major antiTB drugs have emerged. Drug-resistant TB is caused by inconsistent or partial treatment, when patients do not take all their medicines regularly for the required period because they start to feel better, because doctors and health workers prescribe the wrong treatment regimens, or because the drug supply is unreliable. A particularly dangerous form of drug-resistant TB is multidrug-resistant TB (MDR$\mathrm{TB}$ ), which is defined as the disease caused by TB bacilli resistant to at least isoniazid and rifampicin, the two most powerful anti-TB drugs. Rates of MDR-TB are high in some countries, especially in the former Soviet Union, and threaten TB control efforts. While drugresistant TB is generally treatable, it requires extensive chemotherapy (up to two years of treatment) with second-line anti-TB drugs which are more costly than first-line drugs, and which produce adverse drug reactions that are more severe, though manageable.

Quality-assured second-line anti-TB drugs are available at reduced prices for projects approved by the Green Light Committee. One study findings suggest that the current increases in human population, urbanization, and global travel, combined with the population genetic characteristics of $M$. tuberculosis described here, could contribute to the emergence and spread of drug-resistant tuberculosis. ${ }^{29}$ The emergence of extensively drug-resistant (XDR) TB, particularly in settings where many TB patients are also infected with HIV, poses a serious threat to TB control, and confirms the urgent need to strengthen basic TB control and to apply the new WHO guidelines for the programmatic management of drug-resistant TB.

\section{Impact of Imported TB on the Incidence and Prevalence of TB in the Host Country}

The burden of tuberculosis disease is greatest in the developing nations of the world, although the effect of imported disease is observed in low-incidence tuberculosis regions, represented predominantly by high-income countries. In countries such as Canada where TB incidence rate is low, increasing immigration trends may have significant impact on the TB transmission patterns in these countries. ${ }^{30}$ The increase in the rate of TB in the UK, which contrasts with most 
other European countries, may, at least in part, be due to the fact that a high proportion of UK cases occur in the foreign-born, coupled with a comparatively large number of foreign nationals from countries with a very high incidence of TB. ${ }^{31}$ In Madrid, Spain, case rates decreased in association with declining clustered case rates among Spanish-born persons and new strains were introduced through immigration. ${ }^{32}$ In one study in the United States, among the foreign-born population overall, TB case rates declined with increasing time since US entry, but remained higher than among US-born persons-even more than 20 years after arrival. In total, $53 \%$ of TB cases among foreign-born persons occurred among the $22 \%$ of the foreign-born population born in sub-Saharan Africa and Southeast Asia. ${ }^{33}$ Recent studies suggest a re-emergence of tuberculosis among foreign-born children in the State of Florida as from 1997 after several years of decline. The prevalence and incidence rate for TB remains high among immigrants and refugees despite standard pre-immigration screening for TB. TB Screening consists of a PPD skin test for TB, chest X-ray for persons aged 15 years and older; those with chest $\mathrm{X}$-ray suggestive of TB must have their sputum tested for acid-fast bacilli. Applicants who report TB symptoms must also submit specimens of sputum for testing. People are subsequently categorized as:

i. Class A, having TB (X-ray suggestive with at least one sputum positive for acid-fast bacilli),

ii. Class B (X-ray suggestive of active TB, but all 3 sputum specimen negative for acid-fast bacilli,

iii. Class B2 (Positive TB skin test, X-ray suggestive of inactive TB), iv. Having no TB.

In order to travel to the US class A applicants must be treated until sputum specimens are negative for acid-bacilli. Persons with inactive or suspected TB are allowed to travel to the US and local health departments are notified of their arrival by the CDC Division of Global Migration and Quarantine for the follow-up of their evaluation. ${ }^{34}$ One reason for these high TB rates among immigrants is the low sensitivity of the pre-screening tests estimated to detect less than $35 \%$ of TB cases. ${ }^{35}$ There is a need for a more in-depth investigation to elucidate and address causes responsible for the resurgence if the current battle to eliminate tuberculosis from the State is to succeed. National tuberculosis control and elimination programs are increasingly challenged to address disease in foreignborn residents. One study findings indicate that the disease does not disappear and becomes endemic in host areas. ${ }^{36}$ This finding is also supported by numerical simulations with the extended model. The study suggests that immigrants have a considerable influence on the overall transmission dynamics behavior of tuberculosis. The importation of active TB is only a minor part of the total TB burden.

While effective border control is essential, equally, if not more important, are the circumstances that promote the reactivation of latent TB infection in migrant communities, including migrants' experiences in transit and after arrival, structural conditions, and personal characteristics. For sound prevention strategies, attention needs to be paid to the existence of transnational communities and the conditions for migrants, rather than placing a singular focus on place of birth. ${ }^{37}$ Immigration policies and shifting migration patterns over the past 5 decades have brought larger numbers of permanent and temporary residency migrants from high-prevalence regions of the world into low tuberculosis incidence environments. As a consequence, both national immigration policies and global health strategies for the control of tuberculosis share common interest in mobile populations moving from high-to-low prevalence regions. Existing immigration medical screening practices in major immigrant-receiving nations were often designed to prevent and manage the importation of contagious, active pulmonary tuberculosis disease. Such programs may be limited in addressing the long-term consequences of latent tuberculosis infection in foreign-born residents. In nations with a low incidence of tuberculosis, a direct link can be found between the globalization of health factors related to international population movements, as observed with tuberculosis and immigration policies and practices. Continued migration from high-endemic tuberculosis regions will increasingly influence the disease burden in low-endemic areas, and challenge local tuberculosis control and elimination programs. To prevent future increase of transmission of TB, the current control strategies of low-incidence countries are adequate but must be maintained. ${ }^{38}$ Twelve years of $M$. tuberculosis importation as a result of immigration from high-incidence countries had little influence on the transmission of this pathogen in the receiving lowincidence country because measures have been taken to control the problem. Screening at intake to the US Immigration and Customs Enforcement (ICE) custody has helped DIHS staff in diagnosing TB and starting patients on treatment, but patients are usually deported before completing therapy ${ }^{39}$ Because of deportation, and sometimes re-entry into the United States, collaborations are required to support completion of treatment.

\section{Acknowledgements}

None.

\section{Conflict of interest}

Author declares that there is no conflict of interest.

\section{References}

1. World Health Organization. Global Tuberculosis Control. Switzerland; 2009. p. 1-314.

2. CDC. Decrease in reported tuberculosis cases-United States, 2009. Morbidity and Mortality Weekly Report. 2010;59(10):289-294.

3. Daniel TM. The history of tuberculosis. Respir Med. 2006;100(11):18621870.

4. Dye C, Lonnroth K, Jaramillo E, et al. Trends in tuberculosis incidence and their determinants in 134 countries. Bull World Health Organ. 2009;87(9):683-691.

5. Nerlich AG, Haas CJ, Zink A, et al. Molecular evidence for tuberculosis in an ancient Egyptian mummy. Lancet 1997;350(9088): 1404.

6. Taylor GM, Murphy E, Hopkins R, et al. First report of Mycobacterium bovis DNA in human remains from the Iron Age. Microbiology. 2007;153(4):1243-1249.

7. Roguin A. Rene Theophile Hyacinthe Laennec (1781-1826): the man behind the stethoscope. Clin Med Res. 2006;4(3):230-235.

8. Budd W. Memorandum on the nature and the mode of propagation of phthisis. Lancet. 1867;90(2302):451-452.

9. Koch R. Die aetiologie der tuberculose, a translation by Berna Pinner and Max Pinner with an introduction by Allen K Krause. Am Rev Tuberc. $1932 ; 25: 285-323$

10. Von Pirquet C. Frequency of tuberculosis in childhood. JAMA. 1909;52(9):675-678. 
11. Brewer TF, Colditz GA. Relationship between bacille Calmette-Guerin (BCG) strains and the efficacy of BCG vaccine in the prevention of tuberculosis. Clin Infect Dis. 1995;20(1):126-135.

12. Keshavjee S, Farmer PE. Tuberculosis, drug resistance, and the history of modern medicine. N Engl J Med. 2012;367(10):931-936.

13. Ryan KJ, Ray CG. Sherris Medical Microbiology. 4th ed. USA: McGraw Hill-Medical; 2004. p. 1-1088.

14. Baker M, Das D, Venugopal K, Howden-Chapman P. Tuberculosis associated with household crowding in a developed country. J Epidemiol Community Health. 2008;62(8):715-721.

15. World Health Organization. Tuberculosis facts. Switzerland; 2007. p. $1-2$.

16. Dye C, Lonnroth $\mathrm{K}$, Jaramillo E, et al. Trends in tuberculosis incidence and their determinants in 134 countries. Bull World Health Organ. 2009;87(9):683-691.

17. World Health Organization. Global tuberculosis control:a short update to the 2009 report. Switzerland; 2009. p. 1-48.

18. Serpa JA, Teeter LD, Musser JM, et al. Tuberculosis disparity between US-born blacks and whites, Houston, Texas, US. Emerg Infect Dis. 2009;15(6):899-904.

19. CDC. Reported tuberculosis in the United States, 2008a. National Prevention Information Network, USA; 2009.

20. CDC. CDC immigration requirements:Technical instructions for tuberculosis screening and treatment. Georgia; 2009. p. 1-40.

21. CDC. Trends in tuberculosis-United States, 2008b. Morbidity and Mortality Weekly Report. 2009;58(10):249-253.

22. CDC. Trends in tuberculosis-United States, 2007c. Morbidity and Mortality Weekly Report. 2008;57(11):281-285.

23. Lopez De Fede A, Stewart JE, Harris MJ, et al. Tuberculosis in socioeconomically deprived neighborhoods: missed opportunities for prevention. Int J Tuberc Lung Dis. 2008;12(12):1425-1430.

24. Tuberculosis in Prisons. Switzerland: World Health Organization; 2009.

25. Bogorodskaia EM, Ol'khovatskii EM, Borisov SE. Legal aspects of compulsory hospitalization of incompliant patients with tuberculosis. Probl Tuberk Bolezn Legk. 2009;4:8-14.
26. Stanford University. Current Diagnostic Techniques. Mycobacterium Tuberculosis: Novel Approaches to TB Diagnosis, USA; 2009.

27. Vinay Kumar, Abul K Abbas, Nelson Fausto, et al. Robbins Basic Pathology. 8th ed. USA: Saunders Elsevier; 2007. p. 1-960.

28. CDC. Latent tuberculosis infection: a guide for primary health care providers. Georgia; 2013. p. 1-40.

29. Hershberg R, Lipatov M, Small PM, et al. High functional diversity in Mycobacterium tuberculosis driven by genetic drift and human demography. PLoS Biol. 2008;6(12):e311.

30. Zhou Y, Khan K, Feng Z, et al. Projection of tuberculosis incidence with increasing immigration trends. J Theor Biol. 2008;254(2):215-228.

31. Gilbert RL, Antoine D, French CE, et al. The impact of immigration on tuberculosis rates in the United Kingdom compared with other European countries. Int J Tuberc Lung Dis. 2009;13(5):645-651.

32. Inigo J, Arce A, Palenque E, et al. Decreased tuberculosis incidence and declining clustered case rates, Madrid. Emerg Infect Dis. 2008;14(10):641-643.

33. Cain KP, Benoit SR, Winston CA, et al. Tuberculosis among foreignborn persons in the United States. JAMA. 2008;300(4):405-412.

34. Maloney SA, Fielding KL, Laserson KF, et al. Assessing the performance of overseas tuberculosis screening programs:a study among US-bound immigrants in Vietnam. Arch Intern Med. 2006;166(2):234-240.

35. Oeltmann JE, Varma JK, Ortega L, et al. Multidrug-resistant tuberculosis outbreak among US-bound Hmong refugees, Thailand, 2005. Emerg Infect Dis. 2008;14(11):1715-1721.

36. Jia ZW, Tang GY, Jin Z, et al. Modeling the impact of immigration on the epidemiology of tuberculosis. Theor Popul Biol. 2008;73(3):437-448.

37. Littleton J, Park J, Thornley C, et al. Migrants and tuberculosis: analysing epidemiological data with ethnography. Aust $N$ Z J Public Health. 2008;32(2):142-149.

38. Dahle UR, Eldholm V, Winje BA, et al. Impact of immigration on the molecular epidemiology of Mycobacterium tuberculosis in a low-incidence country. Am J Respir Crit Care Med. 2007;176(9):930-935.

39. Schneider DL, Lobato MN. Tuberculosis control among people in US Immigration and Customs Enforcement custody. Am J Prev Med. 2007;33(1):9-14. 